\title{
Stability of ablation flows in inertial confinement fusion: non-modal effects
}

\author{
G. Varillon, ${ }^{1,2}$ J.-M. Clarisse, ${ }^{1}$ and A. Couairon ${ }^{2}$ \\ ${ }^{1}$ CEA, DAM, DIF, F-91297 Arpajon, France \\ ${ }^{2}$ CPHT, CNRS, Ecole Polytechnique, Institut Polytechnique de Paris, Route de Saclay, 91128 Palaiseau, France*
}

(Dated: July 7, 2020)

\begin{abstract}
Fast transient growth of hydrodynamic perturbations due to non-modal effects is shown to be possible in an ablation flow relevant to inertial confinement fusion (ICF). Likely to arise in capsule ablators with material inhomogeneities, such growths appear to be too fast to be detected by existing measurement techniques, cannot be predicted by any of the methods previously used for studying hydrodynamic instabilities in ICF, yet could cause early transitions to nonlinear regimes. These findings call for reconsidering the stability of ICF flows within the framework of non-modal stability theory.
\end{abstract}

Inertial confinement fusion (ICF) has been proposed 50 years ago as a viable means for harnessing thermonuclear fusion with the aim of producing energy. Proceeding from early works on "making the smallest possible fusion explosion" (cf. Ref. 1), ICF relies on implosion flows and inertia forces to bring and maintain fusible elements at required conditions for thermonuclear ignition and burn [2]. This scheme emerged with the advent of lasers as an unprecedented means of irradiating spherical capsules of millimeter size, filled with thermonuclear fuel, at high energy fluxes capable of producing, over nanoseconds, a fuel compression of thousands of times solid density. Such high compressions critically depend, however, on a mitigation of hydrodynamic instabilities during capsule implosion, since these may hamper the compression and heating of the fuel, and possibly ruin the whole process. Since the very beginning of works on the subject, this issue was recognized to be critical for the success of ICF. In particular, the subsonic heat-wave flow, or ablation flow, that results from the irradiation of the outer layer, the ablator, of a fusion capsule and drives its implosion, was right away considered as a dominant source of hydrodynamic perturbation growth. Despite several decades of dedicated numerical simulations, experiments, theoretical works and improvements in the understanding, prediction and mitigation of capsule implosion perturbations, ICF is still in practice impeded by issues of hydrodynamic instability (e.g. Refs. 3, 4).

The strongly compressible, nonuniform and unsteady nature of capsule implosions renders the study of their hydrodynamic stability especially arduous. Linear stability analyses have exclusively consisted in applying the method of normal modes for idealized reduced portions of the implosion flow (e.g. steady, quasi-isobaric, discontinuous ablation flows) or more realistic, i.e. simulated, flows under the frozen-time assumption (cf. Ref. 5 and references therein). Such analyses, however, can only yield asymptotic stability results and are inevitably of restricted validity in time and wavenumber ranges, given the implosion unsteadiness. In fewer instances, dynamical perturbation models capable of accounting for mean flow evolutions, yet in simplified settings, have been used (e.g. Refs. 6, 7). A more global approach, free from such limitations, consists in computing perturbation amplifications about an arbitrary base flow, solution to an initial and boundary value problem (IBVP), as responses to selected initial and/or boundary perturbations. This approach, sometimes called amplification theory (AT), has been largely applied in ICF, especially with multi-dimensional hydrodynamics codes dedicated to ICF physics when simulating experiments, including capsule implosions. Good agreements between AT computations and specifically designed experiments where a dominant, most dangerous, perturbation source is selected by carefully controlling experimental conditions, have helped building confidence in the ability of such codes to reproduce instability dynamics (e.g. Refs. 8, 9).

However AT computations, carried out with these very codes, still display unexplained discrepancies with ablation experiments on capsule ablators at standard specifications for fusion $[10,11]$. In such experiments, many perturbation sources are competing, without a clear dominance of one on the others, and the characterization of their initial or temporal contributions is insufficient for setting up representative enough AT computations. The matter is further complicated by the fact that even individually decaying perturbations, thus held innocuous, can induce, through their interaction, perturbation transient growth [12]. In principle, AT computations could capture such growth provided that they are started from appropriate initial conditions. Yet, performing AT computations for a sufficiently large set of eligible initial or boundary conditions so as to identify those leading to perturbation amplification, and, above all, to the maximum amplification, is unfeasible. Therefore a genuine risk exists of missing the most detrimental instabilities due to a lack of a proper methodology.

In this Letter, we show that non-modal stability theory which exploits the fact that the short-time dynamics of a system is not only ruled by the eigenvalues of its evolution operator but also by this operator eigenfunctions [13], sheds new light on the evolution of hydrodynamic instabilites in ICF. Elaborated over the last thirty years, this theory has been successful in elucidating some withstand- 
ing problems in hydrodynamic stability [14] but had yet to be applied to the stability of ICF flows. This Letter reports results from a first linear non-modal analysis, local in space and time, of an ablation flow relevant to ICF with clear evidences of non-modal effects.

The present stability analysis is conducted on a selfsimilar ablation flow in slab symmetry representative of the early stage of an ICF capsule implosion [15-18]. Such a flow presents the essential features of the deflagration wave that prevails within the capsule ablator during this stage of the implosion (compressibility, stratification, unsteadiness), including its complete structure (Fig. 1): (i) a leading shock front, (ii) a quasi-isentropic compression ('postshock') region, (iii) an ablation layer, and (iv) an expansion wave where heat conduction dominates ('conduction region'). A dimensionless formulation of the equations of motion $[17,19]$ is retained so as to keep the flow description as general as possible. For onedimensional motion along the $x$-axis of a Cartesian coordinate system $(O, x, y, z)$, the equations of motion, written in the Lagrangian coordinate $m$ where $\mathrm{d} m=\rho \mathrm{d} x$, come as

$$
\begin{gathered}
\partial_{t}(1 / \rho)-\partial_{m} v_{x}=0, \quad \partial_{t} v_{x}+\partial_{m} p=0, \\
\partial_{t}\left(\mathrm{C}_{v} T+v_{x}^{2} / 2\right)+\partial_{m}\left(p v_{x}+\varphi_{x}\right)=0,
\end{gathered}
$$

where $\rho, v_{x}, p, T, \varphi_{x}$ denote, respectively, the fluid density, velocity, pressure, temperature and heat flux as functions of $(m, t)$. This system is closed by the dimensionless equation of state for a polytropic gas, $p=\rho T$, with $\mathrm{C}_{v}=1 /(\gamma-1)$ where $\gamma$ is the fluid adiabatic exponent, along with the heat flux $\varphi_{x}=-\rho^{-\mu} T^{\nu} \rho \partial_{m} T$, $\mu \geq 0, \nu>1$. Self-similar reductions of Eq. (1) arise when a semi-infinite slab $(m \geq 0)$, initially such that $\left(\rho, v_{x}, T\right)=(1,0,0)$, is subject to boundary conditions, at the material surface $m=0$, of the form: $\varphi_{x}(0, t)=\mathcal{B}_{\phi} t^{3 \alpha-3}, p(0, t)=\mathcal{B}_{p} t^{2 \alpha-2}$, for $t \geq 0$, with $\alpha=(2 \nu-1) /(2 \nu-2)$. For the choice $\gamma=5 / 3,(\mu, \nu)=$ $(2,13 / 2)$, Eq. (1) describes the motion of a monatomic gas with the radiative conduction model of Kramers [20]. This modeling is an approximation for the ablation of a fusion capsule ablator by hohlraum $\mathrm{x}$ rays in current ICF laser facilities. This approximation is relevant to the ablator opaque portion which stays at temperatures below a few $10^{6}$ Kelvin degrees and at thermodynamic equilibrium. Radiation diffusive effects then dominate those of thermal conduction and viscosity, and radiation pressure and energy are negligible in front of their material counterparts. If Eq. (1) cannot render non-gray irradiation effects, it contains the basic diffusion mechanism at stake, at the hydrodynamic scale, in this ablation process. The particular solution $\left(\mathcal{B}_{\phi}, \mathcal{B}_{p}\right)=(0.8,0.31)$ is chosen for its main features that are typical of the early stage of a capsule implosion [18]: subsonic ablation Mach number, high $(>80)$ ablation front Froude number, steep ablation front, and fast expansion flow with ChapmanJouguet point (Fig. 1). A high accuracy computation

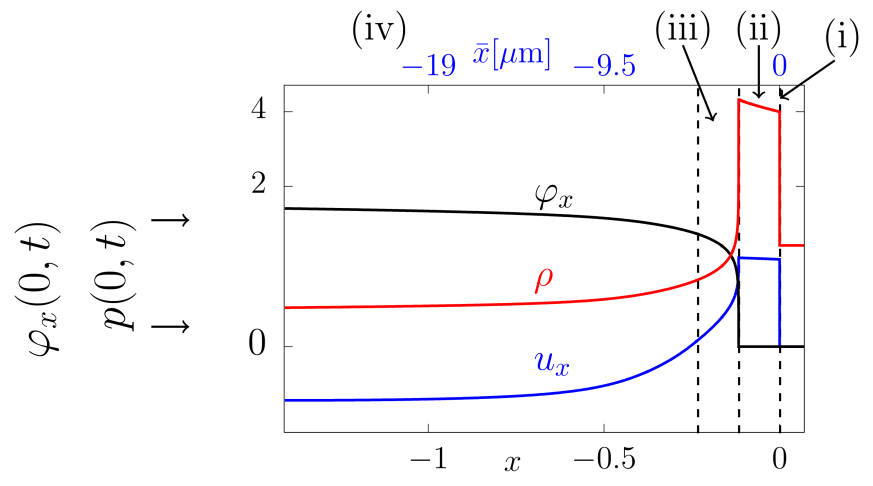

FIG. 1: Self-similar ablation-wave solution to Eq. (1) for $\gamma=5 / 3,(\mu, \nu)=(2,13 / 2)$ and boundary condition parameters $\left(\mathcal{B}_{\phi}, \mathcal{B}_{p}\right)=(0.8,0.31)$. Dimensionless spatial profiles in the coordinate $x$ at time $t_{0}=1$ of the fluid density $\rho$, longitudinal velocity $v_{x}$ and heat flux $\varphi_{x}$. Correspondence with the actual physical extent of the wave, relative to the shock front, at the chosen reference time of capsule implosion ${ }^{a}$ is also indicated (top axis).

${ }^{a}$ See Supplemental Material, Sec. II.

of this flow, down to its finest scales, is provided by an adaptive multidomain Chebyshev method [21].

The stability of flows ruled by Eq. (1) is studied using an Eulerian description of three-dimensional linear perturbations in the coordinate system $(x, y, z)$. Once expressed with the Lagrangian coordinate $m$ and Fourier transformed in the variables $(y, z)$, the corresponding system of governing partial differential equations (Eq. 4 in Ref. [22]) reads in vector form

$$
\partial_{t} \widehat{\mathbf{U}}=\mathcal{A}\left(m, t, \partial_{m}, k_{\perp}\right) \widehat{\mathbf{U}}, \quad \widehat{\mathbf{U}}=\left(\widehat{\rho} \widehat{v}_{x} \widehat{d}_{\perp} \widehat{T}\right)^{\top},
$$

for the $y z$-Fourier components of wavenumber $k_{\perp}=$ $\sqrt{k_{y}^{2}+k_{z}^{2}}: \widehat{f}$, of the perturbations of the base flow variables $f=\rho, v_{x}, T$, and $\widehat{d}_{\perp}$, of the transverse divergence of the transverse velocity perturbation. The perturbation evolution operator, $\mathcal{A}$, depends on space, time and the wavenumber $k_{\perp}$. Perturbation boundary conditions at the geometrically deformed external surface (es) $m=0$ and shock front (sf) $m=m_{\mathrm{sf}}(t)$ are supplementing this system. Solutions to associated IBVPs are computed, in space, using the same multidomain pseudospectral method as for Eq. (1) and, in time, with a three-step implicit-explicit Runge-Kutta scheme.

Modal stability analysis infers the stability of a dynamical system, ruled by an equation like Eq. (2), from the sole basis of the least stable eigenvalue of its evolution operator, $\mathcal{A}$. This analysis is correct if the eigenfunctions of $\mathcal{A}$ form an orthogonal set-equivalently, if the operator $\mathcal{A}$ is normal - and is otherwise only indicative of the long-time behavior of the system. For short-time horizons, a non-normal operator $\mathcal{A}$ may induce transient growth of the system state variable through eigenfunc- 
tion interactions, even if the system is stable according to modal analysis $[13,14][26]$.

The possibility of perturbation short-time growth for the self-similar ablation wave of Fig. 1 is investigated by means of a local analysis, in time and space, of Eq. (2). In effect, for some reference time $t_{0}>0$ and at any location $x_{*}=x\left(m_{*}, t_{0}\right)$ within the wave extent, we consider perturbations of longitudinal characteristic lengths that are shorter than the smallest local gradient length of the flow, say $l_{\nabla}\left(x_{*}\right)$. Under this assumption the operator $\left.\mathcal{A}\right|_{t_{0}}$ may be held as uniform over a neighborhood of $x_{*}$, reducing the analysis of Eq. (2) to that of its Fourier transform in the variable $m$, under the condition $\varkappa_{x}\left(x_{*}\right) \equiv \rho\left(x_{*}\right) k_{m} l_{\nabla}\left(x_{*}\right) \gg 1$ bearing on the longitudinal wavenumber $k_{m}$. Perturbation transient growth is then assessed by computing the maximum instantaneous growth rate $\sigma_{0}\left(k_{m}, k_{\perp}\right)$ [26] of a global norm of $\widehat{\mathbf{U}}$ [27], i.e.

$$
\left.\sigma_{0}\left(k_{m}, k_{\perp}\right) \equiv \max _{\widetilde{\mathbf{U}}_{*}}\left(\frac{1}{\left\|\widetilde{\mathbf{U}}_{*}\right\|^{2}} \frac{\mathrm{d}\left\|\widetilde{\mathbf{U}}_{*}\right\|^{2}}{\mathrm{~d} t}\right)\right|_{t_{0}},
$$

where $\widetilde{\mathbf{U}}_{*}$ stands for the $m$-Fourier component of the restriction of $\widehat{\mathbf{U}}$ to a neighborhood of $m_{*}$. Such computations [23] are carried out for $m_{*}$ covering the whole extent of the ablation wave, for ranges of $k_{m}$ such that $\varkappa_{x}\left(x_{*}\right) \geq 10$, and for different values of $k_{\perp}$. Maps of $\sigma_{0}$ as a function of the flow location $x_{*}$ and of the normalized longitudinal wavenumber $\varkappa_{x}$ are thus obtained (Fig. 2). Regions of non-modal growth, i.e. regions of modal stability but with positive $\sigma_{0}$ [28], are identified (colored areas in Fig. 2) and distinguished from regions of modal instability [29] (black areas). Sizable portions of the conduction region $(-1.15 \lesssim x \lesssim-0.12)$, the ablation layer $(x \approx-0.12)$, for extended ranges of $\varkappa_{x}$, and the postshock region $(-0.12<x<0)$, for restricted $\varkappa_{x}$, are prone to non-modal growth. Maximum growth rates increase with a longitudinal wavelength reduction and non-modal growth is enhanced at shorter transverse wavelengths, both in terms of $\sigma_{0}$ and ranges of $\varkappa_{x}$. This analysis shows that locally the perturbation evolution operator for an ablation wave driven by nonlinear heat conduction is clearly non normal for a wide range of perturbation characteristic lengths. This finding implies that the method of normal modes is insufficient for assessing the stability property of such a flow and that perturbation transient amplifications have to be taken into account and therefore searched for.

The actual occurrence of non-modal growth for the ablation wave of Fig. 1 is confirmed by means of AT computations of $\widehat{\mathbf{U}}$ by solving Eq. (2) for $t \geq t_{0}=1$ with the aforementioned boundary conditions at the external surface and shock front. Initial conditions, inferred from the above non-modal stability analysis, are defined as

$$
\widehat{\mathbf{U}}\left(m, t_{0}, k_{\perp}\right)=w\left(m, k_{m}\right) \operatorname{Re}\left(\widetilde{\mathbf{U}}_{0}^{\text {opt }}\left(m_{*}, k_{m}, k_{\perp}\right) e^{i k_{m} m}\right),
$$

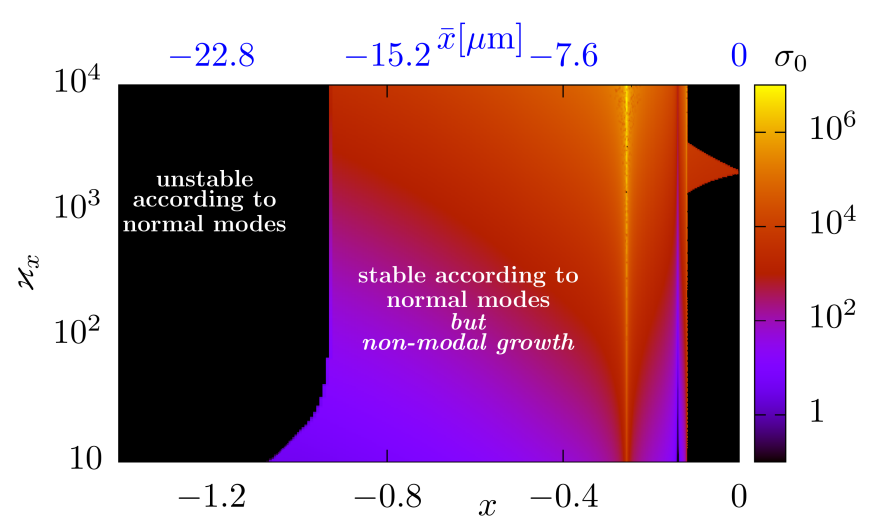

FIG. 2: Intensity map, in the plane $\left(x, \varkappa_{x}\right)$, of $\log \sigma_{0}, \sigma_{0}>0$, obtained from Eq. (3) for $k_{\perp}=1.2$ : regions of non-modal growth but modal stability (color) and regions of modal instability (black). Same horizontal axis conventions as in Fig. 1.

where $w$ is a specifically chosen mask function, sufficiently smooth and non-zero over a limited domain centered about $m=m_{*}$. The function $\widetilde{\mathbf{U}}_{0}^{\text {opt }}$ is the principal eigenfunction of the operator $\left(\mathcal{A}_{0}+\mathcal{A}_{0}^{\dagger}\right), \mathcal{A}_{0}^{\dagger}$ denoting the adjoint operator of $\mathcal{A}_{0}$-i.e. the optimal-growth initial condition yielding the maximum growth rate (3). Results obtained at a location within the post-shock region for normalized wavenumbers $\varkappa_{x}, \varkappa_{\perp}=k_{\perp} l_{\nabla}\left(x_{*}\right)$ for which the method of normal mode predicts stability, are exemplified in Table I(a) and illustrated in Figs. 3 and 4. Growth rates $\sigma_{\mathrm{AT}}$, extracted from AT computations with initial conditions (4), are in good agreement with the values of $\sigma_{0}\left(k_{m}, k_{\perp}\right)$ given by Eq. (3). This agreement and the fact that characteristic growth times, $\sigma_{\mathrm{AT}}^{-1}$, are much smaller than the base-flow characteristic time, here $t \approx 1$, validate the frozen-time assumption and the local analysis leading to Eq. (3). Through these simulations, initial transient growth is verified at flow locations where modal stability analysis predicts decaying perturbations, thus substantiating the reality of local non-modal effects in an elementary ablation flow.

TABLE I: (a) Characteristic growth times: $\sigma_{0}^{-1}$ 'predicted' via Eq. (3) for the flow location $x_{*}=-0.06$, and $\sigma_{\mathrm{AT}}^{-1}$ extracted from AT computations. (b) Corresponding values obtained for the chosen reference time of capsule implosion ${ }^{a}$ at the equivalent location, $1.1 \mu \mathrm{m}$ downstream to the shock front.

(a)

\begin{tabular}{lccc}
\hline \hline$\left(\varkappa_{\perp}, \varkappa_{x}\right)$ & $(1.40,1758)$. & $(5.26,1758)$. & $(131.5,1758)$. \\
\hline$\sigma_{0}^{-1}\left(10^{-5}\right)$ & 15.7 & 15.7 & 8.06 \\
$\sigma_{\mathrm{AT}}^{-1}\left(10^{-5}\right)$ & 15.2 & 15.2 & 7.87 \\
\hline \hline \multicolumn{4}{c}{$(\mathrm{b})$} \\
\hline \hline$\left(\lambda_{\perp}, \lambda_{x}\right)(\mu \mathrm{m})$ & $(100 ., 0.080)$ & $(26.6,0.080)$ & $(1.06,0.080)$ \\
\hline$\sigma_{\mathrm{AT}}^{-1}(\mathrm{ps})$ & 0.22 & 0.22 & 0.11 \\
\hline \hline
\end{tabular}

${ }^{a}$ See Supplemental Material, Sec. II. 
Corresponding data obtained in connection with a chosen ICF capsule implosion [30] are given in Table I(b). The associated figures should be considered as indicative of characteristic lengths of perturbations that are susceptible to yield transient growth. The perturbations presently identified are in the tens of nanometers in the longitudinal (or radial) direction and within the range 1-100 microns in the transverse (or azimuthal) direction, corresponding for the chosen capsule to Legendre modes ranging from 60 to 6000 . These features are, for example, fully compatible with characteristic sizes of material inhomogeneities found in high density carbon ablators [11]. Characteristic growth times $\sigma_{\mathrm{AT}}^{-1}$ are in the sub-picosecond range, i.e. way below the time resolutions of existing measurement techniques of flow modulations in ICF ablation experiments, making the direct detection of such fast dynamics most unlikely in practice. This initial growth, characterized by an amplification by more than six of the perturbation norm (Fig. 3), results from the constructive interaction between the localized entropy and acoustic waves that dominate the optimal-growth initial condition (4): see Figs. 4(a-d). The ensuing perturbation dynamics reflects further mu-

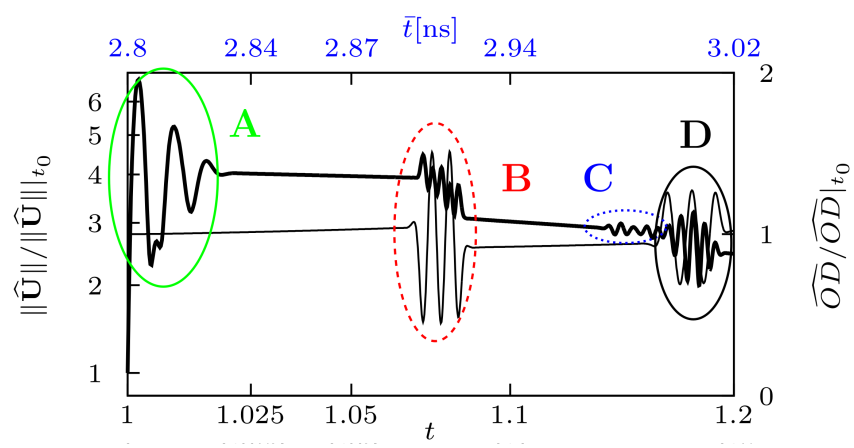

FIG. 3: Amplification of the perturbation norm, $\|\widehat{\mathbf{U}}\| /\left.\|\widehat{\mathbf{U}}\|\right|_{t_{0}}$, (thick line - left axis) and of the optical depth perturbation, $\widehat{\mathrm{OD}} /\left.\widehat{\mathrm{OD}}\right|_{t_{0}}$ (thin line-right axis) for the case $\left(\varkappa_{x}, \varkappa_{\perp}\right)=$ (1758., 1.40). Remarkable events in the evolution of $\|\widehat{\mathbf{U}}\|$ are identified by letters A to D.

tual interactions of these waves [events A and C, Figs. 3 and 4(a-c)], propagation [Figs. 4(b,c,d)], and interactions with the ablation layer [event B, Figs. 3 and $4(\mathrm{a}, \mathrm{b}, \mathrm{d})$ ] and shock front [event D, Figs. 3 and $4(\mathrm{a}, \mathrm{b})]$. The optical depth perturbation [31] turns out to be insensitive to the transient growth, displaying variations only for the perturbation interactions with the ablation layer and shock front (events B and D). In the conduction region, local constructive interaction has also been found to induce transient growth. Hence constructive interaction between localized compressible fluid waves comes out from the present analysis as a mechanism of initial transient growth for the current ablation wave modeling. Furthermore, the constructive interaction evidenced here is likely to arise in actual ablation flows since ablator ma- terial inhomogeneities trigger acoustic and entropy emissions from both the shock front and the ablation layer.
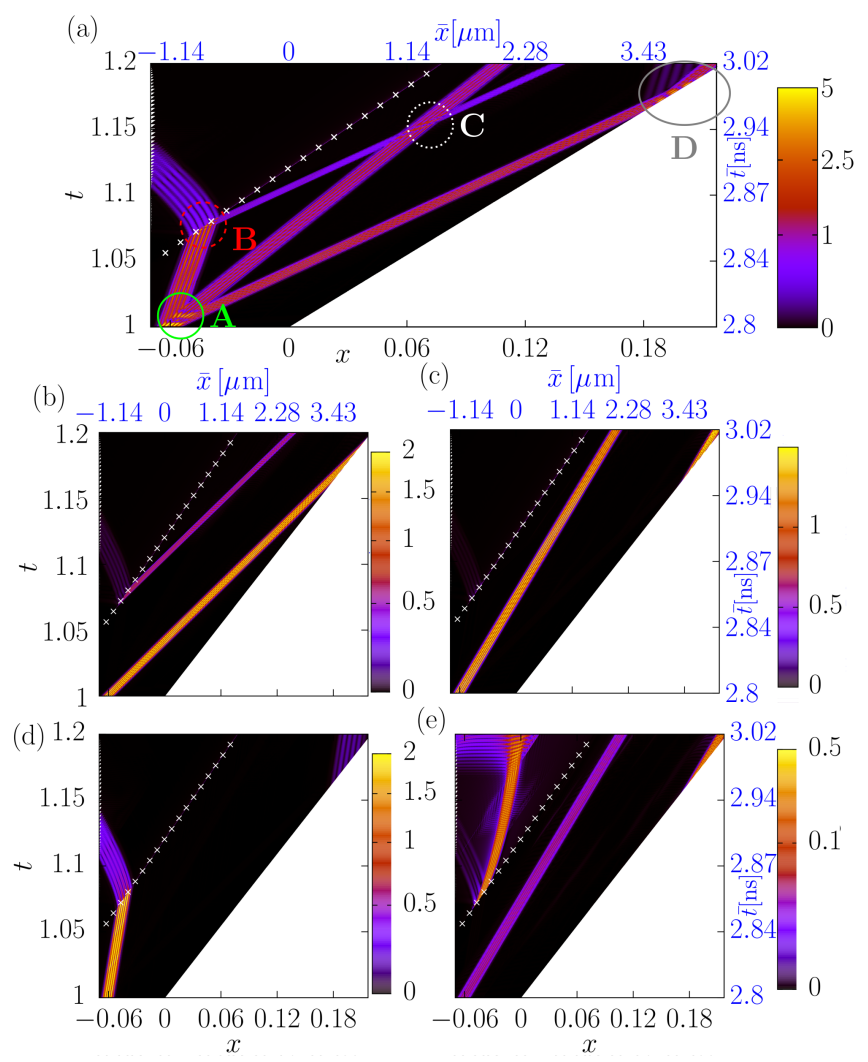

FIG. 4: Perturbation $\widehat{\mathbf{U}}$ in the post-shock region as function of the variables $(x, t)$, for initial conditions given by Eq. (4) in the case $\left(\varkappa_{x}, \varkappa_{\perp}\right)=(1758,1.40)$. (a) Local Euclidean norm. Components (absolute value) in longitudinal pseudo-characteristic variables (cf. Ref. [22]): (b) forward and (d) backward acoustic waves, (c) entropy waves. (e) Transverse potential vorticity. Labelled circles in (a) relate to the events identified in Fig. 3.

This Letter brings the first evidences that non-modal effects play a role in the stability of an ablation flow related to ICF capsule implosions. In effect, local perturbation transient growth is found to occur for wide ranges of characteristic lengths in flow regions that are stable according to the classical method of normal modes. The identified mechanism of transient growth - the constructive interaction of compressible fluid waves - is intrinsic to compressible fluid motion and thus generic to actual ablation flows. In the cases put forth, transient growth happens over time scales that are beyond current experimental capabilities and without direct impact on optical depth signal. Hence fast amplifications, capable of inducing transitions to instability nonlinear regimes earlier than foreseen by modal stability analysis, can actually happen while remaining undetected. These findings stress the necessity of performing global non-modal stability analyses of ablation flows in ICF so as to establish 
on firm grounds the prediction of their hydrodynamic instabilities. Such analyses, despite the more involved and demanding methods of non-modal stability theory [24] that they require, are worth undertaking given their potential benefits for the success of ICF.

* gregoire.varillon@polytechnique.edu

[1] J. Lindl, Phys. Plasmas 2, 3933 (1995), URL https:// doi.org/10.1063/1.871025.

[2] S. Atzeni and J. Meyer-ter-Vehn, The physics of inertial fusion (Oxford University Press, Oxford, U.K., 2004).

[3] J. Lindl et al., Phys. Plasmas 21, 020501 (2014).

[4] B. M. Haines et al., Phys. Plasmas 26, 012707 (2019).

[5] V. Bychkov et al., Prog. Energy Combust. Sci. 47, 32 (2015).

[6] V. N. Goncharov et al., Phys. Plasmas 7, 2062 (2000).

[7] P. Amendt et al., Phys. Plasmas 10 (2003).

[8] Y. Aglitskiy et al., Phil. Trans. R. Soc. A 368, 1739 (2010).

[9] K. S. Raman et al., Phys. Plasmas 21 (2014).

[10] V. A. Smalyuk et al., Phys. Plasmas 22, 072704 (2015).

[11] S. J. Ali et al., Phys. Plasmas 25 (2018).

[12] P. J. Schmid and D. S. Henningson, Stability and transition in shear flows (Springer, 2001).

[13] L. N. Trefethen et al., Science 261, 578 (1993).

[14] P. J. Schmid, Annu. Rev. Fluid Mech. 39, 129 (2007).

[15] R. Marshak, Phys. Fluids 1, 24 (1958).
[16] F. Abéguilé et al., Phys. Rev. Lett. 97, 035002 (2006).

[17] C. Boudesocque-Dubois et al., J. Fluid Mech. 603, 151 (2008).

[18] J.-M. Clarisse et al., J. Fluid Mech. 848, 219 (2018).

[19] J.-M. Clarisse et al., J. Fluid Mech. 609, 1 (2008).

[20] Y. B. Zel'dovich and Y. P. Raizer, Physics of shock waves and high-temperature hydrodynamic phenomena (Academic Press, New York, 1967).

[21] C. Boudesocque-Dubois et al., J. Comput. Phys. 235, 723 (2013).

[22] G. Varillon et al., Phys. Rev. E 101, 043215 (2020).

[23] C. C. Cowen and E. Harel, An effective algorithm for calculating the numerical range (1995), URL https://www . math. iupui.edu/ ccowen/Downloads/33NumRange.pdf.

[24] P. Luchini and A. Bottaro, Annu. Rev. Fluid Mech. 46, 493 (2014).

[25] D. Mihalas and B. W. Mihalas, Foundations of radiation hydrodynamics (Oxford University Press, Oxford, 1984).

[26] See Supplemental Material, Sec. I.

[27] Here, the chosen global perturbation norm is defined after the scalar product $\langle\widehat{\mathbf{U}}, \widehat{\mathbf{V}}\rangle=\frac{1}{2} \int_{0}^{m_{\mathrm{sf}}} \rho^{-1} \widehat{\mathbf{U}}^{\dagger} \widehat{\mathbf{V}} \mathrm{d} m$.

[28] See Supplemental Material, Fig. 1(c).

[29] See Supplemental Material, Fig. 1(b).

[30] See Supplemental Material, Sec. II.

[31] Hydrodynamic disturbances are classically detected in ICF flows through measurements of optical depth perturbations [9] , here $\widehat{\mathrm{OD}}=\int_{0}^{m_{\mathrm{sf}}} \rho^{-1}\left(\kappa_{\rho} \widehat{\rho}+\kappa_{T} \widehat{T}\right) \mathrm{d} m+$ $\left[\partial_{x} \kappa \widehat{x}\right]_{\text {es }}^{\text {sf }}$, where $\kappa=4 \rho^{\mu} T^{3-\nu}$ is the fluid opacity [25], and $\widehat{x}_{\mathrm{es} \mid \mathrm{sf}}$, the deformation of the external surface|shock front. 